\title{
Foramen Vertebral Lumbar Inconstante en Bos taurus
}

\author{
Inconstant Lumbar Vertebral Foramen in Bos taurus
}

\author{
Martin Lima; William Pérez
}

\begin{abstract}
LIMA, M. \& PÉREZ, W. Foramen vertebral lumbar inconstante en Bos taurus. Int. J. Morphol., 25(4):895-898, 2007.
RESUMEN: En Anatomía Veterinaria la investigación de los forámenes de la columna vertebral es necesaria para expandir nuestro conocimiento de osteología sistemática, y para el estudio de la vascularización de la columna vertebral y de la médula espinal. El objetivo de este trabajo es informar acerca de la presencia y características de forámenes inconstantes hallados en las vértebras lumbares bovinas. Quince de 100 (15\%) vértebras lumbares presentaban forámenes. Todos los forámenes estaban localizados en el cuerpo de la vértebra lumbar sobre su cara lateral derecha. Cuatro de estas vértebras eran la lumbar 2 y tres de ellas la lumbar 5.
\end{abstract}

\section{PALABRAS CLAVE: Anatomía; Huesos; Forámenes inconstantes; Cavidad ósea.}

\section{INTRODUCCIÓN}

De acuerdo a Smuts (1974) información detallada sobre la presencia y distribución de forámenes nutricios y de otro tipo, sobre las caras externa y vertebral de las vértebras de los animales domésticos parece faltar. Publicaciones recientes sobre la irrigación sanguínea de los animales domésticos no consideran forámenes como los descritos en este trabajo.

Un estudio detallado de los forámenes de la columna vertebral no sólo llenará un vacío en nuestros conocimientos de osteología, sino que también es prerrequisito para el estudio de la irrigación sanguínea de la columna vertebral y de la médula espinal.

Forámenes inconstantes no descritos en los libros de texto clásicos de Anatomía veterinaria fueron recientemente descritos en la cabeza ósea bovina (Rinderknecht et al., 2005) y un foramen supratroclear en el húmero fue descrito en 3 bovinos adultos de Turquía (Haziroglu \& Ozer, 1990).

En este caso nosotros reportamos la presencia de forámenes óseos inconstantes localizados en vértebras lumbares bovinas. Hasta donde se conoce, no existe referencia con respecto a estos forámenes en la bibliografía veterinaria. Ni los libros de texto de Anatomía (Barone, 1999; Nickel, 1977; Sisson, 1975) o Patología (Jubb, Kennedy \& Palmer, 1993) hacen referencia a su existencia, tampoco se encontró ninguna publicación científica que los describa. No obstante, únicamente Barone (1996) cita en general forámenes de la cara ventral de las vértebras donde emergen venas basivertebrales, pero no indica la ubicación, las vértebras en que aparecen, el tamaño de estos forámenes ni tampoco, como ya se indicó antes, los describe en su libro de Osteología (Barone, 1999).

Por lo tanto, el objetivo del presente trabajo es informar sobre la presencia y características de los forámenes inconstantes en vértebras lumbares bovinas.

\section{MATERIAL Y MÉTODO}

Cien vértebras lumbares fueron estudiadas. Treinta vértebras correspondían a 5 esqueletos completos, por lo cual se conocía para cada una su ubicación exacta. Las demás sólo fueron diagnosticadas como vértebras lumbares bovinas y por no ser totalmente seguro, no se determinó su ubicación exacta. Estos huesos pertenecían a animales adultos de raza Holstein (Bos taurus) de edad superior a 5 años pertenecientes al Museo de Anatomía de la Facultad de Veterinaria, Uruguay.

Se estudiaron la presencia de forámenes inconstantes, el número de forámenes por vértebra, el diámetro de los mismos y la forma de cada uno. Se tomarón fotografías con cámara digital Nikon. 


\section{RESULTADOS}

El 15\% de las vértebras lumbares bovinas presentaban forámenes. Estos forámenes estaban localizados en el cuerpo de las vértebras lumbares sobre su cara lateral derecha (Fig. 1). En la cara lateral izquierda del cuerpo vertebral en caso de estar presente algún foramen era de tamaño menor a 1,0 mm (Fig. 2), nunca tan desarrollado como en la cara derecha.

El foramen del lado derecho comunicaba con un canal óseo que se abría sobre la cara dorsal del cuerpo de la vértebra separado por un delgado tabique óseo dando el aspecto de ser un agujero doble (Fig. 3).

El tamaño y la forma del foramen derecho eran variables, los diámetros craneocaudal y dorsoventral en relación a la longituddel cuerpo vertebral aparecen en la Tabla I.

El foramen derecho inconstante tenía forma subcircular, elíptica, o algunas veces más irregular.

En dos de cinco esqueletos completos los forámenes derechos estaban localizados en las vértebras lumbares 2 y 5 , en dos solamente en la lumbar 2 (Fig. 4), y en uno de los esqueletos sólo en la lumbar 5.

Tabla I. Tamaño y diámetros craneocaudal y dorsoventral del foramen vertebral lumbar derecho en relación a la longitud del cuerpo vertebral. $(\mathrm{cm})$

\begin{tabular}{ccc}
\hline L.C.V. & D.C.C. & D. D. V. \\
\hline 6,9 & 1,5 & 0,9 \\
6 & 3 & 3 \\
6 & 1,9 & 0,6 \\
6 & 1,4 & 0,8 \\
4,5 & 0,2 & 0,2 \\
6,5 & 1,3 & 0,4 \\
5 & 0,8 & 0,6 \\
5 & 0,3 & 0,1 \\
5,5 & 0,8 & 0,5 \\
5,5 & 1 & 0,6 \\
7 & 2 & 1,3 \\
6,6 & 1,1 & 0,6 \\
5 & 1 & 0,8 \\
5,2 & 1,2 & 0,7 \\
6 & 1,2 & 0,8 \\
\hline
\end{tabular}

L.C.V.: Largo del cuerpo vertebral.

D.C.C.: Diámetro craneocaudal del foramen derecho.

D.D.V: Diámetro dorsoventral del foramen derecho.

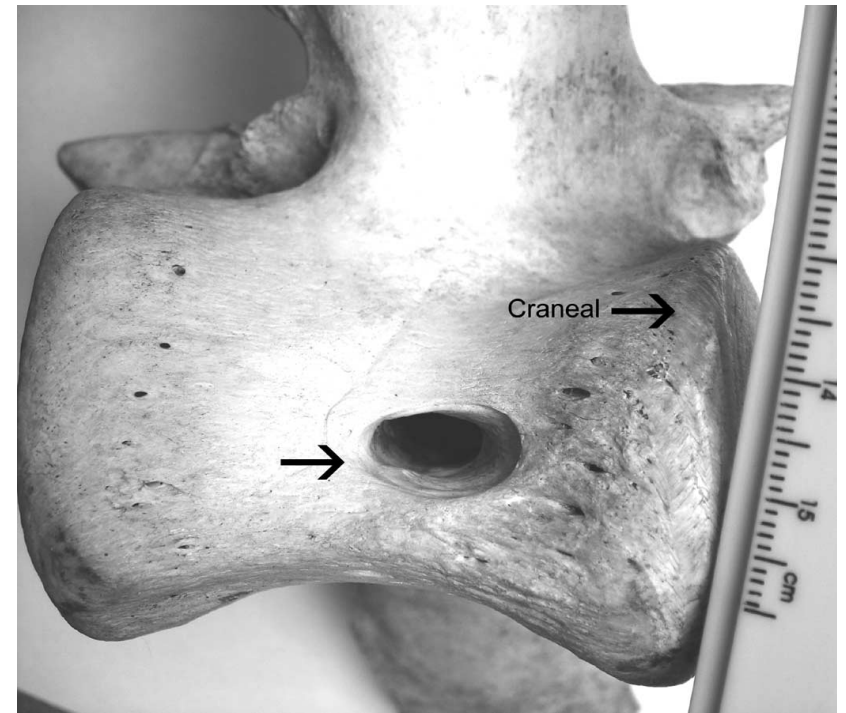

Fig. 1. Lado derecho del cuerpo de una vértebra lumbar bovina. La flecha indica el foramen vertebral lumbar.

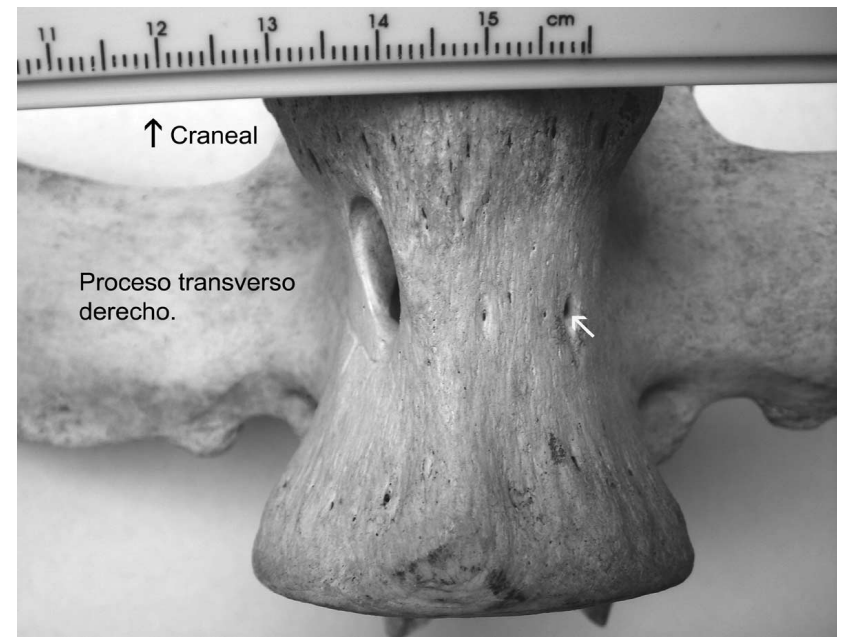

Fig. 2. Vista ventral del cuerpo de la vértebra anterior.

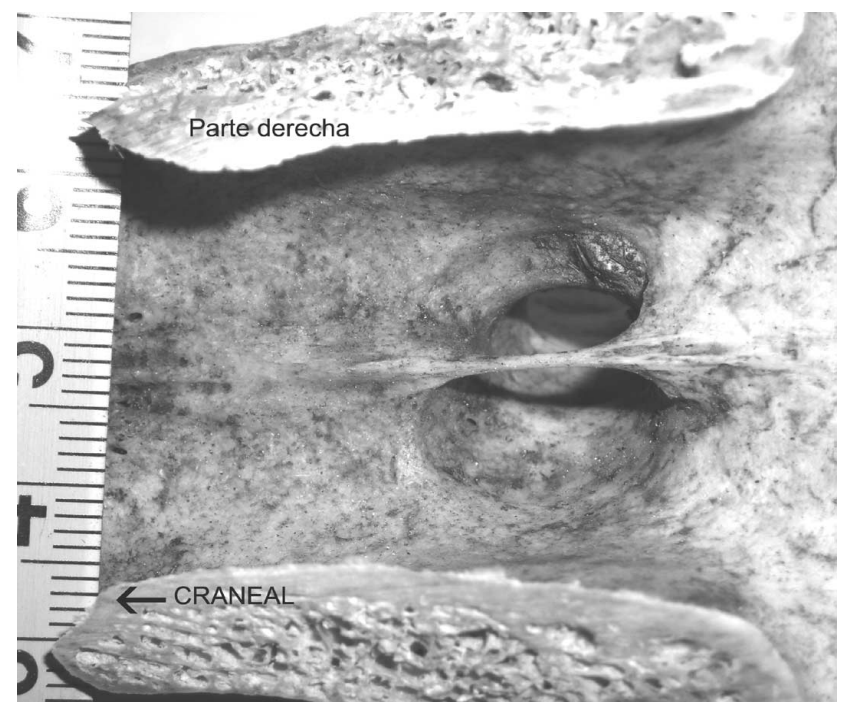

Fig. 3. Vista dorsal del cuerpo vertebral tras remoción del arco. 


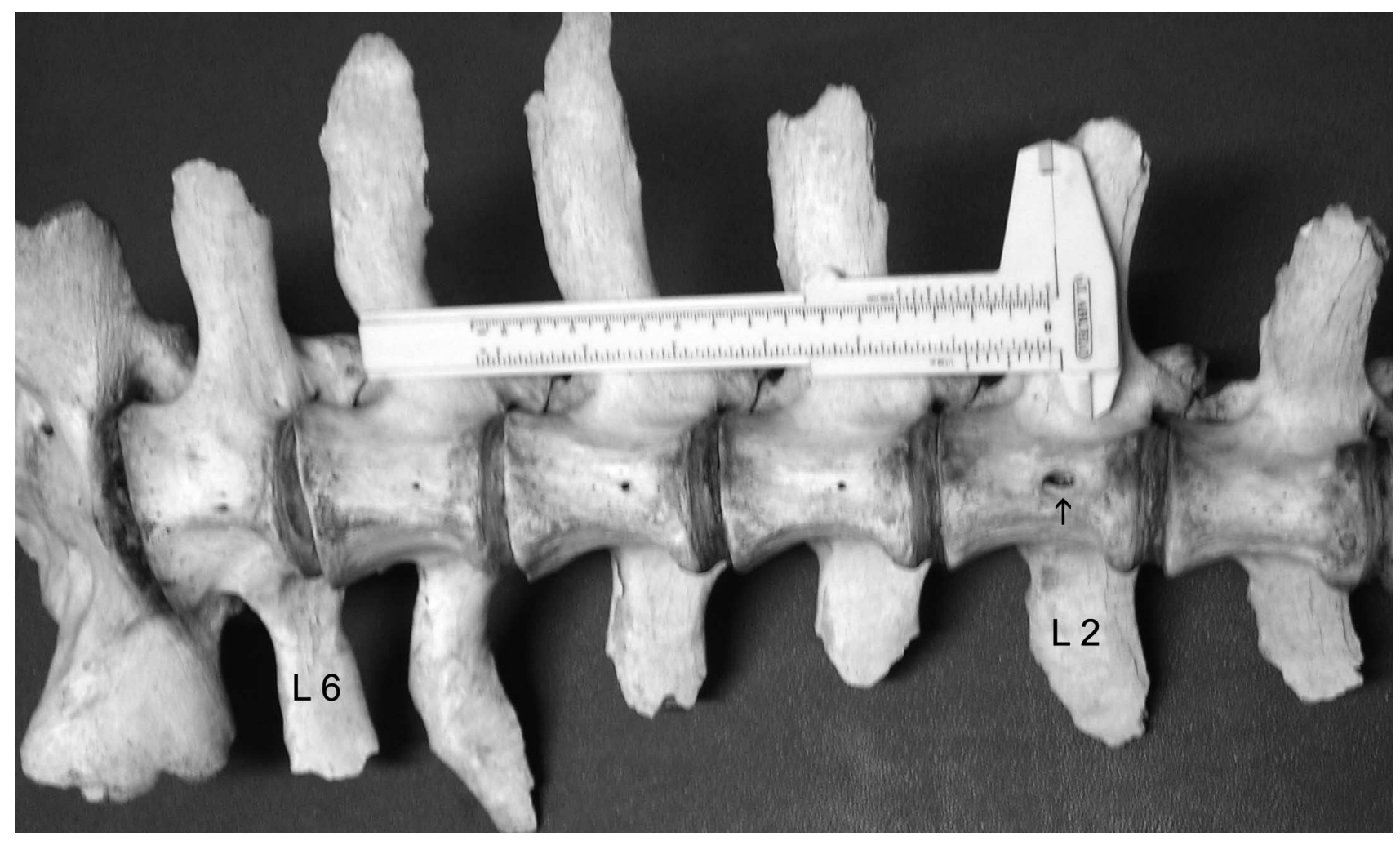

Fig. 4. Vista ventral derecha de vértebras lumbares con el foramen vertebral en la lumbar 2 (flecha).

\section{DISCUSIÓN}

En este caso se informa sobre la presencia de forámenes inconstantes en el cuerpo de las vértebras lumbares bovinas. Estos forámenes parecen estar mayormente localizados en las vértebras lumbar 2 y 5 , pero en la mayoría de las vértebras estudiadas por ser aisladas no se pudo determinar con exactitud su número.

Estos forámenes pueden estar relacionados a vasos sanguíneos que salen de las vértebras como los vasos basivertebrales que se unen al plexo venoso vertebral externo en la cara ventral de las vértebras (Barone, 1996). Es necesario investigar mediante diversas técnicas la vascularización de las vértebras lumbares en orden de correlacionar con los forámenes descritos en este trabajo. Lo interesante a dilucidar es por qué estos forámenes aparecieron en algunas vértebras y sólo del lado derecho.

LIMA, M. \& PÉREZ, W. Foramen vertebral lumbar inconstante en Bos taurus. Int. J. Morphol., 25(4):895-898, 2007.

SUMMARY: In Veterinary Anatomy the research of the foramina of the vertebral column is necessary for to expand our knowledge of systematic osteology, and for study of the vascularization of the vertebral column and spinal cord. The objective of this work is to inform about presence and characteristics of inconstant foramina found in bovine lumbar vertebrae. Fifteen of 100 (15\%) of lumbar vertebrae presented foramina. All foramina were found at the body of lumbar vertebrae on their right lateral face. Four of these vertebrae were lumbar 2 and three of them lumbar 5.

KEY WORDS: anatomy, bones, inconstant foramina, osseous cavity. 


\section{REFERENCIAS}

Barone, R. Anatomie Comparée des Mammifères Domestiques. $1^{\text {th }}$ Ed. Angiologie. Vigot Frères, Paris, 1996. V. 5.

Barone, R. Anatomie Comparée des Mammifères Domestiques. $4^{\text {th }}$ Ed. Osteologie. Vigot Frères, Paris, 1999. V. 1.

Haziroglu, R. M. \& Ozer, M. A Supratrochlear Foramen in the Humerus of Cattle. Anat. Histol. Embryol., 19:1068,1990 .

Jubb, K. V. F.; Kennedy, P. C. \& Palmer, N. Pathology of Domestic Animals. $4^{\text {th }}$ Ed. Academic Press, 1993. V. 1.

Nickel, R. A.; Schummer, E.; Seiferle, J. F. \& Wille, K. H. 1977. Lehrbuch der Anatomie 12 der Haustiere. Bewegungsapparat, I. Paul Parey, Berlin.

Rinderknecht, A.; Pérez, W.; Bielli, A. \& Möller, R. Inconstant skull foramina in bovine (Bos taurus) and swine (Sus scrofa). J. of Animal and Veterinary Advances, 4 (9): 798-800, 2005.

Sisson, S. Ruminant Osteology. In: Sisson and Grossman's the Anatomy of the Domestic Animals. (R. Getty, ed.) $5^{\text {th }}$ ed. W. B. Saunders, Philadelphia. V. 1. pp. 741-786, 1975 .

Smuts, M. M. S. The Foramina of the Cervical Vertebrae of the Ox. Part I: Atlas and Axis. Anatomia, Histologia, Embryologia. J. of Veterinary Medicine Series C 3 (4):296-307, 1974.

Smuts, M. M. S. The Foramina of the cervical vertebrae of the Ox Part II: Cervical Vertebrae 3-7. Anatomia, Histologia, Embryologia. J. of Veterinary Medicine Series $C 4$ (1):24-37, 1975.
Dirección para correspondencia:

Dr. William Pérez

Área de Anatomía

Facultad de Veterinaria

Universidad de la República

Lasplaces 1620

11600 Montevideo

URUGUAY

Email: vetanat@gmail.com

Recibido : 24-08-2007

Aceptado: 29-09-2007 